\title{
The association of ectopic craniopharyngioma in the fourth ventricle with familial adenomatous polyposis: illustrative case
}

\author{
Hiroya Uemura, MD, ${ }^{1}$ Masahiro Tanji, MD, PhD, ${ }^{1}$ Hiroki Natsuhara, MD, ${ }^{1}$ Yasuhide Takeuchi, MD, $\mathrm{PhD},{ }^{2}$ Masahito Hoki, MD, ${ }^{2}$ \\ Akihiko Sugimoto, $\mathrm{MD},{ }^{2}$ Sachiko Minamiguchi, MD, PhD, ${ }^{2}$ Hidenori Kawasaki, MD, PhD, ${ }^{3}$ Masako Torishima, $\mathrm{PhD},{ }^{3}$ \\ Shinji Kosugi, MD, PhD, ${ }^{4}$ Yohei Mineharu, MD, PhD, ${ }^{1}$ Yoshiki Arakawa, MD, PhD, ${ }^{1}$ Kazumichi Yoshida, MD, PhD, ${ }^{1}$ and \\ Susumu Miyamoto, MD, $\mathrm{PhD}^{1}$
}

\footnotetext{
${ }^{1}$ Department of Neurosurgery, Kyoto University Graduate School of Medicine, Kyoto, Japan; ${ }^{2}$ Department of Diagnostic Pathology, Kyoto University Hospital, Kyoto, Japan; and Departments of ${ }^{3}$ Genomic Medicine and ${ }^{4}$ Medical Ethics and Medical Genetics, Kyoto University School of Public Health, Kyoto, Japan
}

BACKGROUND Craniopharyngioma (CP) often arises in the sellar and suprasellar areas; ectopic CP in the posterior fossa is rare. Familial adenomatous polyposis (FAP) is a genetic disorder involving the formation of numerous adenomatous polyps in the gastrointestinal tract, and it is associated with other extraintestinal manifestations.

OBSERVATIONS The authors reported the case of a 63-year-old woman with FAP who presented with headache and harbored a growing mass in the fourth ventricle. Magnetic resonance imaging (MRI) findings revealed a well-circumscribed mass with high intensity on T1-weighted images and low intensity on T2-weighted images and exhibited no contrast enhancement. Gross total resection was performed and histopathology revealed an adamantinomatous $\mathrm{CP}(\mathrm{aCP})$. The authors also reviewed the previous reports of ectopic $\mathrm{CP}$ in the posterior fossa and found a high percentage of FAP cases among the ectopic CP group, thus suggesting a possible association between the two diseases.

LESSONS An ectopic CP may be reasonably included in the differential diagnosis in patients with FAP who present with well-circumscribed tumors in the posterior fossa.

https://thejns.org/doi/abs/10.3171/CASE21572

KEYWORDS craniopharyngioma; familial adenomatous polyposis; fourth ventricle; $\beta$-catenin

Craniopharyngioma $(\mathrm{CP})$ is a benign tumor derived from the embryonic tissue of the sellar region and its surrounding structures. $^{1}$ There are two types of CP: adamantinomatous CP (aCP) and papillary $C P(p C P)$. The aCP type arises from the cells of the embryonic structure, known as the craniopharyngeal duct whereas pCP arises from the cells of the anterior part of the pituitary gland. ${ }^{1}$ CPs usually grow in the sellar-suprasellar area. An ectopic CP arising from other sites is rare; $\mathrm{CP}$ of the posterior fossa is even rarer and has only been reported in 19 cases so far. ${ }^{2,3}$ Moreover, there have only been five reports of $\mathrm{CP}$ arising from the fourth ventricle. $^{4,5}$
Familial adenomatous polyposis (FAP) is an autosomal dominant genetic disorder in which adenomatous polyps form in the epithelium of the gastrointestinal tract. The disorder is caused by germline mutations in the adenomatous polyposis coli $(A P C)$ gene, which is a tumor suppressor. ${ }^{6}$ FAP is also associated with other extraintestinal manifestations, such as gastric fundic gland polyps, duodenal polyps, pancreatobiliary tumors, congenital hypertrophy of retinal pigment epithelium, fibromas, fibromatosis, nasal angiofibromas, thyroid carcinomas, hepatoblastomas, and brain tumors. ${ }^{6}$

Herein, we report a case of CP located purely in the fourth ventricle in a patient with FAP. We also review the previous literature

ABBREVIATIONS aCP = adamantinomatous $\mathrm{CP} ; \mathrm{CP}=$ craniopharyngioma; $\mathrm{FAP}=$ familial adenomatous polyposis; $\mathrm{MRI}=$ magnetic resonance imaging; $\mathrm{pCP}=$ papillary CP.

INCLUDE WHEN CITING Published January 3, 2022; DOI: 10.3171/CASE21572.

SUBMITTED October 9, 2021. ACCEPTED October 16, 2021.

(C) 2022 The authors, CC BY-NC-ND 4.0 (http://creativecommons.org/licenses/by-nc-nd/4.0/). 
on ectopic $\mathrm{CP}$ and discuss the possible relationship between these rare diseases.

\section{Illustrative Case}

A 63-year-old woman with FAP was referred to our department because of a mass in the fourth ventricle that was detected using magnetic resonance imaging (MRI), which was performed because of chronic headaches. She had a known history of FAP and underwent prophylactic colectomy at the age of 32 years. Later, the patient developed rectal cancer, which was resected when she was 57 years old. Apart from the gastrointestinal manifestations, she had frontal bone osteoma and received surgery for its removal at the age of 22 years. Her mother died from colon cancer at the age of 60 years, and her brother had colon cancer in his 30s. Also, her sister had received surgery for sigmoid colon cancer in her 40 s. The target sequence by NCC oncopanel FC v2.0 revealed a truncating frameshift variant in the 15th exon of the germline APC gene (NM_000038.5: c.3180_3184del: p.l1060fs), thus confirming the diagnosis of FAP.

The patient exhibited no neurological deficits, except for chronic headaches. MRI demonstrated a well-circumscribed mass with a maximum diameter of $32 \mathrm{~mm}$ in the fourth ventricle, with heterogeneously high intensity on T1-weighted images and low intensity on T2-weighted images (Fig. $1 \mathrm{~A}$ and B). No contrast enhancement of the mass was observed (Fig. 1C), and no evidence of obstructive hydrocephalus was found. MR angiography showed that the mass was located near the left posterior inferior cerebellar artery; however, digital subtraction angiography showed no evidence of thrombosed aneurysm. Computed tomography showed a small component of calcification inside the mass (Fig. 1D), whereas fluorodeoxyglucose positron emission tomography showed no accumulation in the mass. Based on imaging findings, a preoperative diagnosis of epidermoid, or neurenteric cyst, was made. The patient was followed-up with serial MRI. Eleven months after the first visit, the mass showed a tendency to grow, and the patient agreed to proceed with surgical removal of the mass.

The patient was placed prone, and a midline suboccipital craniotomy was performed under general anesthesia. The mass in the fourth ventricle was cystic (Fig. 2A) and was found to have no connection with the posterior inferior cerebellar artery. Puncture of the cyst revealed a fluid component resembling motor oil. The capsule had no adhesion to the floor of the fourth ventricle, and the cyst was easily dissected and removed en bloc (Fig. 2B).

Hematoxylin and eosin staining revealed that the cyst wall was proliferated with basal cell-like p40-positive squamous epithelial
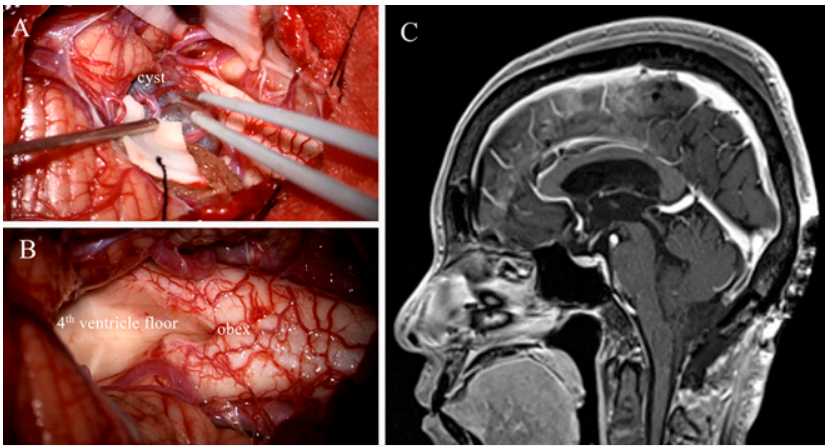

FIG. 2. Intraoperative findings and postoperative MRI. A: The cyst wall was observed to be occupying the fourth ventricle. B: The tumor was removed en bloc without adhesion to the floor of the fourth ventricle. C: Postoperative sagittal T1-CE images showing gross total resection of the tumor.

cells with high nuclear-to-cytoplasmic ratio and was accompanied by wet-keratin (Fig. 3A). The contents were keratinized and characterized by a well-differentiated squamous epithelium with palisading columnar epithelium (Fig. 3B). Immunohistochemistry revealed positive staining for $\beta$-catenin in the membrane and focally in the nucleus (Fig. 3C). The BRAF (V600E) staining results were negative. Overall, the pathological diagnosis was concluded to be aCP.

The patient had an uneventful postoperative recovery, and postoperative MRI revealed complete resection of the tumor (Fig. 2C). At the 2-month follow-up visit, MRI showed no evidence of any recurrence.

\section{Discussion \\ Observations}

$\mathrm{CP}$ is a benign tumor arising from the remnant tissue of the craniopharyngeal canal and is known to be predominantly located in and around the sella turcica, accounting for $3 \%$ of all intracranial tumors. ${ }^{1}$ Clinical symptoms include headaches and vomiting due to increased intracranial pressure and visual field disturbances due to optic nerve compression. Pathologically, there are two types of $\mathrm{CP}$ : aCP and $\mathrm{pCP} .{ }^{1}$ Although the common site of $\mathrm{CP}$ is around the sella turcica of the pituitary stalk, primary ectopic $\mathrm{CP}$ occurring in other locations in the cranium has also been reported, including in the nasopharynx, sphenoid bone, and third ventricle. ${ }^{7-9}$ A total of 19 patients with ectopic $\mathrm{CP}$ in the posterior fossa have been reported
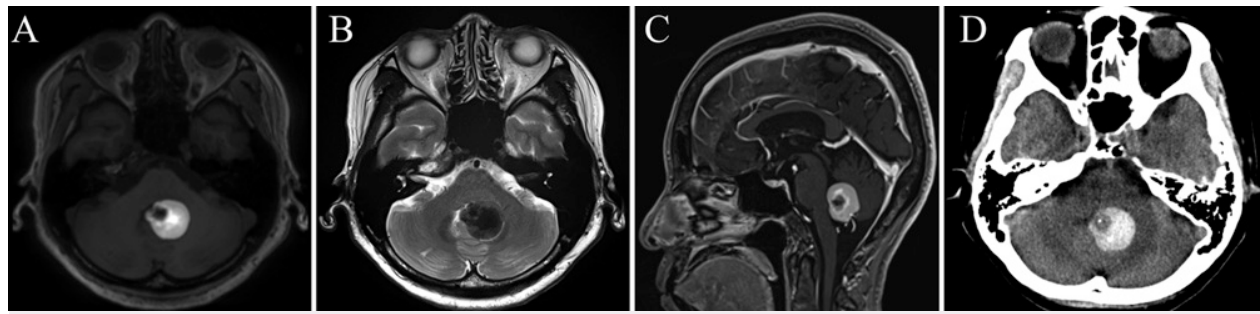

FIG. 1. Preoperative images. A: Axial T1-weighted images showing a hyperdense cystic mass. B: Axial T2weighted images showing a hypodense mass in the fourth ventricle. C: Sagittal T1-contrast enhanced images showing a cystic mass without enhancement. D: Axial computed tomography (CT) images demonstrate small calcifications within the mass. 

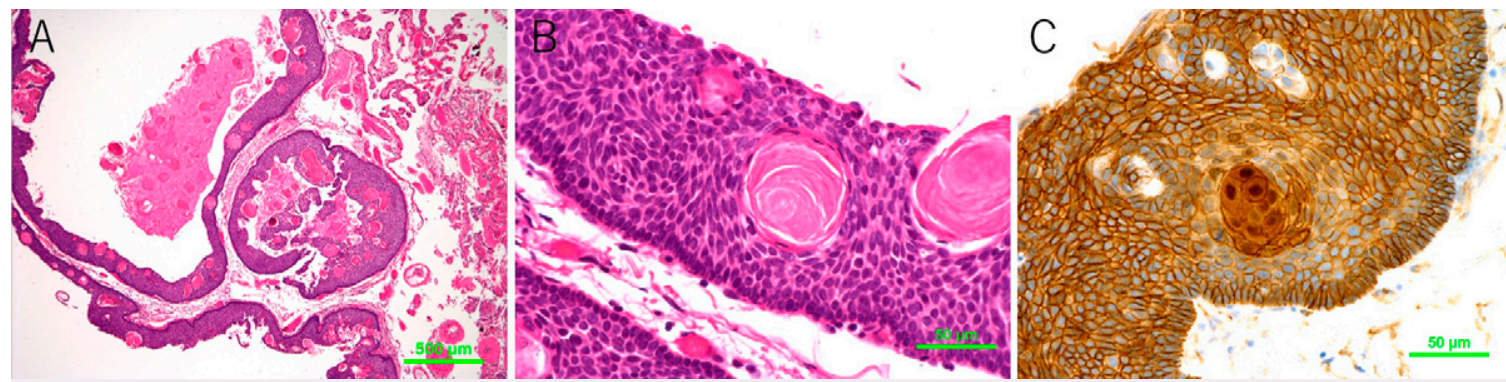

FIG. 3. Histopathology. A: The cystic lesion contained "wet" keratin (hematoxylin and eosin stain). Bar $=500 \mu \mathrm{m}$. B: The well-differentiated squamous epithelium was surrounded by palisading columnar epithelium (hematoxylin and eosin stain). Bar $=50 \mu \mathrm{m}$. C: Immunohistochemistry for $\beta$-catenin showed that some foci of epithelial cells were positive for $\beta$-catenin in their nuclei. Bar $=50 \mu \mathrm{m}$.

to date, of whom 6 patients, including ours, were diagnosed with $\mathrm{CP}$ in the fourth ventricle (Table 1)., ${ }^{2,4,5,10-23}$ The high incidence of FAP among patients with ectopic $\mathrm{CP}$ in the posterior fossa suggests an association between these two rare diseases. Of the 20 cases involving the posterior fossa, 14 were men and 18 were younger than 40 years. The average age of patients with $\mathrm{CP}$ who have FAP (mean [SD] age: 33.9 [13.6] years) was approximately 10 years more than patients without FAP (mean [SD] age: 23.8 [12.0] years). There were no patients younger than 20 years among the patients with FAP, whereas 5 of 13 patients without FAP were younger than 20 years. Our case is unique because it is the first reported case of this condition in a woman; moreover, she had the greatest age at surgery among the patients with FAP. Headache was the most common clinical symptom observed in patients with ectopic $\mathrm{CP}$ of the posterior fossa $(71 \%)$. Diplopia and hearing impairment were also observed in 7 patients $(33 \%)$. All pathological findings were aligned with $\mathrm{aCP}$ in patients with FAP, except for 1 case in which it was not mentioned; however, 2 cases of $\mathrm{pCP}$ were reported in patients without FAP. As for the extent of tumor removal, total resection was performed in 14 cases, whereas near-total and subtotal resection were performed in 3 cases each. Excluding our case, CP with FAP has been reported in 7 patients to date, ${ }^{2-5}$ of whom most (6 of 7 cases) occurred in the posterior fossa. Of the 6 cases of $\mathrm{CP}, 2$ cases that were complicated by FAP occurred in the fourth ventricle.

FAP is an autosomal dominant genetic disorder characterized by multiple adenomatous polyps in the gastrointestinal mucosa. It is also associated with extragastrointestinal lesions and brain tumors, including medulloblastoma, ependymoma, and pituitary adenoma. ${ }^{6}$

TABLE 1. Literature review of ectopic craniopharyngiomas in the posterior fossa

\begin{tabular}{|c|c|c|c|c|c|}
\hline Author \& Year & Age (yrs)/Sex & FAP & Clinical Symptoms & Location & Histopathology \\
\hline Gökalp \& Mertol, $1990^{11}$ & $17 / \mathrm{M}$ & - & Headache, vomiting, hearing loss, ataxia & CPA & $\mathrm{N} / \mathrm{A}$ \\
\hline Xu et al., $2006^{13}$ & $22 / \mathrm{F}$ & - & Tinnitus, dizziness, hearing loss & CPA & $\mathrm{aCP}$ \\
\hline Shah et al., $2007^{14}$ & $12 / \mathrm{F}$ & - & Headache, diplopia, walking disorder & Fourth ventricle & $\mathrm{aCP}$ \\
\hline Yilmaz et al., $2010^{17}$ & $14 / \mathrm{M}$ & - & Rt hearing loss, diplopia, neck pain & CPA & $\mathrm{N} / \mathrm{A}$ \\
\hline Sharma et al., $2012^{18}$ & $26 / \mathrm{F}$ & - & Headache & CPA & $\mathrm{aCP}$ \\
\hline Khalatbari et al., $2012^{19}$ & $40 / M$ & - & Headache, diplopia, tinnitus, It hearing loss, palsy of V, VI & CPA & $\mathrm{aCP}$ \\
\hline Khalatbari et al., $2012^{19}$ & 22/M & - & Headache, vomiting, diplopia, tinnitus, ataxia & CPA & $\mathrm{aCP}$ \\
\hline Bozbuga et al., $2011^{22}$ & $34 / \mathrm{M}$ & + & Hemiparesis, tinnitus, It hearing loss & CPA & $\mathrm{aCP}$ \\
\hline Kim et al., $2014^{23}$ & $31 / \mathrm{M}$ & + & Headache, dizziness & $\mathrm{CPA}$ & $\mathrm{aCP}$ \\
\hline Álvarez Salgado et al., $2017^{4}$ & 29/M & + & Rt facial paresis, diplopia & Fourth ventricle & $\mathrm{aCP}$ \\
\hline Pena et al., $2016^{5}$ & 20/M & + & None & Fourth ventricle & $\mathrm{aCP}$ \\
\hline Present case & $63 / F$ & + & Headache & Fourth ventricle & $\mathrm{aCP}$ \\
\hline
\end{tabular}


Recent studies have correlated the severity of gastrointestinal disease and the prominence of extraintestinal findings to specific mutations within the APC gene. ${ }^{24}$ Patients with FAP who have APC gene mutations between codons 697 and 1224 have a three-fold increased risk of developing brain tumors in general and a 13-fold increased risk of developing medulloblastoma when compared with patients with FAP who have other APC gene mutations. ${ }^{25}$ Among the 7 cases of CP with FAP reported to occur in the posterior fossa, there was only 1 case in which a similar genetic diagnosis was made. $^{20}$ In that case, a truncating frameshift mutation in the APC gene was detected in the 16th exon (NM_000038: c.4541_4547del: p.P1514fs), leading to a stop at the position of the 18th codon.

There are two possible hypotheses that explain the high incidence of FAP among ectopic CP cases in the posterior fossa. First, a report explains the mechanism of primary ectopic CP as possibly being the migration of squamous epithelial cell remnants to the obliterated craniopharyngeal canal. ${ }^{26}$ The craniopharyngeal canal usually disappears during the fetal period. If it is left behind, it is called the Rathke's pouch, and it can be the site of $\mathrm{CP}$, which occurs in close proximity to the pharynx as described above. ${ }^{7-9}$ However, in our case, it was located purely in the fourth ventricle, with no apparent continuity with the pharynx. This suggests that in patients with FAP, squamous cells in the craniopharyngeal canal may migrate to the posterior fossa in the early stages of development by an unknown mechanism or the maldifferentiation of cells in the posterior fossa by germline mutations in the APC gene. Second, it has been reported that mutant $A P C$ proteins are unable to induce the degradation of $\beta$-catenin, resulting in the accumulation of $\beta$-catenin in the cells. ${ }^{27} \beta$-catenin mutation is also found in aCP heterozygotes. ${ }^{24}$ The pathological accumulation of $\beta$-catenin in aCP is known to be associated with FAP, suggesting the possible molecular level association between these diseases. However, compared to medulloblastoma, CPs are less common in patients with FAP, which could be because of the relationship between CP and FAP that has not yet been fully established or because of regulatory elements in the developing epithelium where aCP occurs and nullifies the pathogenicity of APC deficiency. ${ }^{3}$ The precise molecular or cellular mechanism remains unelucidated, and the association between these two diseases is notable, necessitating the need for further studies.

\section{Lessons}

Ectopic CPs in the posterior cranial fossa are rare. However, ectopic CP may be reasonably included in the list of differential diagnoses in patients with FAP who present with well-circumscribed tumors in the posterior fossa, especially in the fourth ventricle.

\section{References}

1. Müller $\mathrm{HL}$. The diagnosis and treatment of craniopharyngioma. Neuroendocrinology. 2020;110(9-10):753-766.

2. Algahtani AY, Algahtani HA, Jamjoom AB, Samkari AM, Marzuk YI. De novo craniopharyngioma of the fourth ventricle: case report and review of literature. Asian J Neurosurg. 2018;13(1):62-65.

3. Dahl NA, Pratt D, Camelo-Piragua S, et al. Pediatric craniopharyngioma in association with familial adenomatous polyposis. Fam Cancer. 2019;18(3):327-330.

4. Álvarez Salgado JA, González-Llanos Fernández de Mesa F, Villaseñor Ledezma JJ, et al. Ectopic craniopharyngioma and Gardner's syndrome: case report and literature review. Article in Spanish. Neurocirugia (Astur). 2017;28(2):97-101.
5. Pena AH, Chaudhry A, Seidman RJ, Peyster R, Bangiyev L. Ectopic craniopharyngioma of the fourth ventricle in a patient with Gardner syndrome. Clin Imaging. 2016;40(2):232-236.

6. Dinarvand P, Davaro EP, Doan JV, et al. Familial adenomatous polyposis syndrome: an update and review of extraintestinal manifestations. Arch Pathol Lab Med. 2019;143(11):1382-1398.

7. Kundu S, Dewan K, Varshney H, Das S, Mukhopadhyay S, Ghosh A. An atypical rare case of extracranial craniopharyngioma. Indian J Otolaryngol Head Neck Surg. 2014;66(1):122-125.

8. Cooper PR, Ransohoff J. Craniopharyngioma originating in the sphenoid bone. Case report. J Neurosurg. 1972;36(1):102-106.

9. Rambarki O, Rajesh A. Third ventricular craniopharyngioma. Neurol India. 2016;64(4):834-835.

10. Altinörs N, Senveli E, Erdoğan A, Arda N, Pak I. Craniopharyngioma of the cerebellopontine angle. Case report. J Neurosurg. 1984;60(4):842-844.

11. Gökalp HZ, Mertol T. Cerebellopontine angle craniopharyngioma. Neurochirurgia (Stuttg). 1990;33(1):20-21.

12. Bashir EM, Lewis PD, Edwards MR. Posterior fast craniopharyngioma. Br J Neurosurg. 1996;10(6):613-615.

13. Xu F, Liu X, Xu ZA. A case of ectopic craniopharyngioma. Chin J Neurosurg. 2006;21:762.

14. Shah GB, Bhaduri AS, Misra BK. Ectopic craniopharyngioma of the fourth ventricle: case report. Surg Neurol. 2007;68(1):96-98.

15. Powers CJ, New KC, McLendon RE, Friedman AH, Fuchs HE. Cerebellopontine angle craniopharyngioma: case report and literature review. Pediatr Neurosurg. 2007;43(2):158-163.

16. Yan Y, Tang WY, Yang G, Zhong D. Isolated cerebellopontine angle craniopharyngioma. J Clin Neurosci. 2009;16(12):1655-1657.

17. Yilmaz C, Altinors N, Sonmez E, Gulsen S, Caner H. Rare lesions of the cerebellopontine angle. Turk Neurosurg. 2010;20(3):390-397.

18. Sharma M, Mally R, Velho V, Hrushikesh K. Primary isolated cerebellopontine angle papillary craniopharyngioma. Neurol India. 2012;60(4):438-439.

19. Khalatbari MR, Borghei-Razavi H, Samadian M, Moharamzad Y, Schick U. Isolated primary craniopharyngioma in the cerebellopontine angle. J Clin Neurosci. 2012;19(11):1516-1519.

20. Link MJ, Driscoll CL, Giannini C. Isolated, giant cerebellopontine angle craniopharyngioma in a patient with Gardner syndrome: case report. Neurosurgery. 2002;51(1):221-226.

21. Aquilina K, O'Brien DF, Farrell MA, Bolger C. Primary cerebellopontine angle craniopharyngioma in a patient with Gardner syndrome. Case report and review of the literature. J Neurosurg. 2006;105(2):330-333.

22. Bozbuga M, Turan Suslu $H$, Hicdonmez T, Bayindir C. Primary cerebellopontine angle craniopharyngioma in a patient with Gardner syndrome. J Clin Neurosci. 2011;18(2):300-301.

23. Kim MS, Kim YS, Lee HK, Lee GJ, Choi CY, Lee CH. Primary intracranial ectopic craniopharyngioma in a patient with probable Gardner's syndrome. J Neurosurg. 2014;120(2):337-341.

24. Lamlum $H$, llyas $M$, Rowan $A$, et al. The type of somatic mutation at $A P C$ in familial adenomatous polyposis is determined by the site of the germline mutation: a new facet to Knudson's 'two-hit' hypothesis. Nat Med. 1999;5(9):1071-1075.

25. Attard TM, Giglio P, Koppula S, Snyder C, Lynch HT. Brain tumors in individuals with familial adenomatous polyposis: a cancer registry experience and pooled case report analysis. Cancer. 2007;109(4):761-766.

26. Kawamata T, Kubo O, Kamikawa S, Hori T. Ectopic clival craniopharyngioma. Acta Neurochir (Wien). 2002;144(11):1221-1224.

27. Krausova M, Korinek V. Wnt signaling in adult intestinal stem cells and cancer. Cell Signal. 2014;26(3):570-579.

\section{Disclosures}

Dr. Kawasaki reported "The genomic medicine course I belong to is an industry-academia joint research course with Konica Minolta. However, 
it has nothing to do with the content of this research." No other disclosures were reported.

\section{Author Contributions}

Conception and design: Tanji, Uemura, Kosugi, Yoshida. Acquisition of data: Tanji, Uemura, Natsuhara, Takeuchi, Sugimoto. Analysis and interpretation of data: Tanji, Uemura, Sugimoto. Drafting the article: Tanji, Uemura, Sugimoto, Kawasaki. Critically revising the article: Tanji, Uemura, Sugimoto, Torishima, Kosugi, Mineharu, Arakawa, Yoshida, Miyamoto.
Reviewed submitted version of manuscript: Uemura, Sugimoto, Minamiguchi, Arakawa. Approved the final version of the manuscript on behalf of all authors: Tanji. Statistical analysis: Uemura. Administrative/ technical/material support: Tanji, Minamiguchi. Study supervision: Tanji, Kawasaki, Yoshida, Miyamoto. Pathology diagnosis: Hoki.

\section{Correspondence}

Masahiro Tanji: Kyoto University Graduate School of Medicine, Kyoto, Japan. tanji@kuhp.kyoto-u.ac.jp. 